Research Article

\title{
Critical Analysis of Stage IV Epithelial Ovarian Cancer Patients after Treatment with Neoadjuvant Chemotherapy followed by Cytoreductive Surgery and Hyperthermic Intraperitoneal Chemotherapy (CRS/HIPEC)
}

\author{
Carlos A. Munoz-Zuluaga $\mathbb{D}^{1},{ }^{1}$ Armando Sardi ${ }^{(D},{ }^{1}$ Michelle Sittig ${ }^{(D)},{ }^{1}$ Vadim Gushchin $\left(\mathbb{D},{ }^{1}\right.$ \\ Mary C. King, ${ }^{1}$ Carol Nieroda $\mathbb{D}^{1},{ }^{1}$ Felipe Lopez-Ramirez $\mathbb{D}$, ${ }^{1}$ and Teresa P. Diaz-Montes $\mathbb{D}^{2}$ \\ ${ }^{1}$ Department of Surgical Oncology, The Institute for Cancer Care at Mercy Medical Center, Baltimore, Maryland 21202, USA \\ ${ }^{2}$ The Lya Segall Ovarian Cancer Institute at Mercy Medical Center, Baltimore, Maryland 21202, USA
}

Correspondence should be addressed to Teresa P. Diaz-Montes; tdiaz@mdmercy.com

Received 30 June 2020; Revised 14 October 2020; Accepted 26 November 2020; Published 17 December 2020

Academic Editor: C. H. Yip

Copyright (c) 2020 Carlos A. Munoz-Zuluaga et al. This is an open access article distributed under the Creative Commons Attribution License, which permits unrestricted use, distribution, and reproduction in any medium, provided the original work is properly cited.

\begin{abstract}
Background. Cytoreductive surgery and hyperthermic intraperitoneal chemotherapy (CRS/HIPEC) after neoadjuvant chemotherapy (NACT) showed promise as initial treatment for stage IIIC (SIII) epithelial ovarian cancer (EOC); however, stage IV (SIV) outcomes are rarely reported. We assessed our experience and outcomes treating newly diagnosed SIV EOC with NACT plus CRS/HIPEC compared to SIII patients. Methods. Advanced EOC from 2015-2018 managed with NACT (carboplatin/ paclitaxel) due to unresectable disease or poor performance status followed by interval CRS/HIPEC were reviewed. Perioperative factors were assessed. Overall survival (OS) and progression-free survival (PFS) were analyzed by stage. Results. Twenty-seven FIGO stage IIIC $(n=12)$ and IV $(n=15)$ patients were reviewed. Median NACT cycles were 3 and 4 , respectively. Post-NACT omental caking, ascites, and pleural effusions decreased/resolved in $91 \%, 91 \%$, and $100 \%$ of SIII and $85 \%$, $92 \%$, and $71 \%$ of SIV. SIII/SIV median PCI was 21 and 20 obtaining $92 \%$ and $100 \%$ complete cytoreduction $(\leq 0.25 \mathrm{~cm})$, respectively. Median organ resections were 6 and 7, respectively. Grade III/IV surgical complications were 0\% SIII and 23\% SIV, without hospital mortality. Median time to adjuvant chemotherapy was 53 and 74 days, respectively $(p=0.007)$. SIII OS at 1 and 2 years was $100 \%$ and $83 \%$ and $87 \%$ and $76 \%$ in SIV $(p=0.269)$. SIII 1 -year PFS was 54\%; median PFS: 12 months. SIV 1- and 2- year PFS was $47 \%$ and $23 \%$; median PFS: 12 months $(p=0.944)$. Conclusion. Outcomes in select initially diagnosed and unresectable SIV EOC are similar to SIII after NACT plus CRS/HIPEC. SIV EOC may benefit from CRS/HIPEC, and further studies should explore this treatment approach.
\end{abstract}

\section{Introduction}

Epithelial ovarian, fallopian tube, and primary peritoneal cancers, known as epithelial ovarian cancer (EOC), are heterogeneous diseases staged and treated similarly $[1,2]$. These diseases account for the majority of deaths from gynecological cancers in developed countries, due to scarcity of symptoms at early stages and lack of screening methods [3]. Consequently, most EOC patients are diagnosed after peritoneal spread (International Federation of Gynecology and Obstetrics (FIGO) stage III/IV) with 5-year survival of $29 \%[3]$.

Primary debulking surgery (PDS) followed by postoperative or adjuvant systemic chemotherapy (ASC) with taxane-platinum combinations is standard for advanced EOC (AEOC) [4]. However, over the past decade, new strategies have been pursued to improve outcomes, including the use of neoadjuvant systemic chemotherapy (NACT) and, more recently, intraoperative hyperthermic intraperitoneal chemotherapy (HIPEC) [5-10]. NACT plus interval 
cytoreductive surgery (CRS) without HIPEC demonstrated improved perioperative outcomes but nonsuperiority in terms of time to recurrence and survivals compared to PDS $[5,11,12]$. Recently, NACT plus interval CRS with HIPEC showed improved survival for stage III patients, but its' role in stage IV patients, who typically have limited treatment options and high mortality, is unclear $[13,14]$. We assessed our experience treating newly diagnosed stage IV (SIV) EOC with NACT plus interval CRS/HIPEC and compared findings to the same treatment cohort of stage IIIC (SIII) patients.

\section{Patients and Methods}

An institutional CRS/HIPEC database was reviewed, identifying newly diagnosed AEOC patients who received NACT followed by CRS/HIPEC from 2015-2018. This treatment approach was offered at our institution to AEOC patients deemed ineligible for the randomized clinical trial (NCT 02124421) which assesses the role of CRS/HIPEC as initial treatment in AEOC. NACT criteria included unresectable peritoneal disease due to extensive small bowel or porta hepatis involvement, biliary obstruction, or encasement of common/ external iliac vessels evidenced by imaging and/or laparoscopy, extra-abdominal disease, Eastern Cooperative Oncology Group (ECOG) performance status $>2$, and/or large volume of ascites or pleural effusion. Staging occurred before NACT and after CRS/HIPEC, assigning the highest staging for analysis.

Three cycles of NACT with systemic taxane/platinum regimens were administered. NACT response and surgical eligibility was evaluated with imaging, tumor markers, and performance status. Interval CRS/HIPEC was offered if complete CRS (residual disease $<0.25 \mathrm{~cm}$ ) was feasible, followed by 3 cycles of taxane/platinum ASC, totaling 6 cycles.

2.1. Response Evaluation. Treatment response was based on CT scan of chest/abdomen/pelvis pre-/post-NACT using Response Evaluation Criteria in Solid Tumors (RECIST) version 1.1 [15]. To note, the noncomplete response/nonprogressive disease (non-CR/non-PD) label is used over stable disease for patients with only nonmeasurable disease in this classification. [15] CA-125 levels were measured preNACT, post-NACT/pre-CRS/HIPEC, post-CRS/HIPEC, and post-ASC. Histopathologic chemotherapy response is not reported due to lack of consensus [16].

\subsection{Interval Cytoreductive Surgery/Hyperthermic Intraperi-} toneal Chemotherapy (CRS/HIPEC) Procedure. Post-NACT, patients were considered CRS/HIPEC candidates if there was no gross extra-abdominal disease, gross resolution of prior pleural effusion, ECOG performance status $\leq 2$, and complete cytoreduction was feasible $(<0.25 \mathrm{~cm}$ residual disease). Intraoperative disease burden was estimated using Peritoneal Cancer Index (PCI) scores [17]. Multiple peritoneal and visceral resections were performed to reduce tumor to microscopic levels. Completeness of cytoreduction (CC) score quantified residual tumor with CC-0 (no visible residual disease) and $\mathrm{CC}-1$ (residual tumor $<0.25 \mathrm{~cm}$ ) considered complete cytoreductions [18]. Incomplete cytoreduction was defined as tumor nodules $0.25 \mathrm{~cm}-2.5 \mathrm{~cm}$ (CC-2) or $>2.5 \mathrm{~cm}$ (CC-3). HIPEC agents included carboplatin $800 \mathrm{mg} / \mathrm{m}^{2}$ ( 90 minutes, $41-43^{\circ} \mathrm{C}$ ) or melphalan $50 \mathrm{mg} /$ $\mathrm{m}^{2}$ (90 minutes, $41-42^{\circ} \mathrm{C}$ ), if platinum resistance was suspected based on NACT response. Bowel anastomoses and chest tube placement were performed following perfusion. All procedures were performed together by an experienced team of gynecologic and surgical oncologists, specializing in peritoneal surface malignancies. Patients were transferred to the intensive care unit (ICU) for 24 hours and then to the inpatient oncology unit when clinically stable. Surgical complications were considered until postoperative day (POD) 90 and graded according to Clavien-Dindo classification with grade III/IV considered major [19].

2.3. Staging. AJCC $8^{\text {th }}$ edition staging was performed before NACT and at CRS/HIPEC using the highest staging for subgroup designation [20]. Stage IV disease included positive pleural effusion cytology (pM1a or FIGO stage IVA), liver or splenic parenchymal metastases, extra-abdominal metastases, including inguinal and extra-abdominal lymph nodes, and/or transmural intestinal involvement (cM1b/ pM1b or FIGO stage IVB).

2.4. Follow-Up. Postoperative follow-up occurred 3 and 6 weeks after discharge, every 3 months for 2 years, and every 6 months thereafter, for 5 years. After ASC, CT scan of chest/ abdomen/pelvis were performed every 6 months or, as clinically indicated. After complete cytoreduction (CC-0/1), disease recurrence was defined by radiographic/pathologic evidence or disease.

2.5. Statistical Analysis. Categorical variables were analyzed using chi-squared or Fisher's exact test and continuous variables using independent sample Student's $t$ test or MannWhitney $U$ test, when not normally distributed. Survival analysis was performed using KaplanMeier method and logrank test. Overall survival (OS) was calculated from CRS/ HIPEC to date of death. Progression-free survival (PFS) was calculated from CRS/HIPEC to date of radiographic/pathologic disease recurrence, or date of death from disease, whichever occurred first. PFS was only calculated with complete cytoreduction (CC-0/1). Median follow-up time was estimated using the reverse KaplanMeier method. All analyses were performed using STATA version 12.0 (StataCorp LLC, College Station, TX, USA) and considered statistically significant if $p \leq 0.05$.

2.6. Ethics. IRB approval and preoperative consent were obtained.

\section{Results}

Thirty AEOC patients received NACT from January 2015-December 2018. Three (10\%) were not surgical candidates for CRS/HIPEC due to progression through NACT with unresectable disease $(n=2)$ and failure to thrive $(n=1)$. Two of these patients died 7.9 and 10.6 months from 
diagnosis and 4.9 and 8.6 months after completing NACT. The other patient was lost to follow-up with an unknown status. Twenty-seven patients received interval CRS/ HIPEC: 12, stage IIIC (SIII) and 15, stage IVA/B (SIV) (Figure 1 and Table 1). Distant metastases in SIV included extra-abdominal metastases (inguinal and extra-abdominal lymph nodes) in 7 (47\%), positive pleural effusion cytology in $5(33 \%)$, transmural intestinal involvement in $2(13 \%)$, and splenic parenchymal metastasis in $1(7 \%)$ patient.

3.1. Response to Neoadjuvant Systemic Chemotherapy. According to RECIST 1.1 criteria, [15] 12 patients (SIII = 5/ SIV $=7,44 \%$ ) had measurable disease (lymph nodes, masses) and 15 (SIII $=7 / \mathrm{SIV}=8,56 \%)$ had only nonmeasurable disease (mesenteric caking, effusions) after NACT. Complete response $(\mathrm{CR})$ was seen in $4(\mathrm{SIII}=1 / \mathrm{SIV}=3,15 \%)$, partial response in 4 $(\mathrm{SIII}=2 / \mathrm{SIV}=2,15 \%)$, stable disease in $6(\mathrm{SIII}=3 / \mathrm{SIV}=3$, $22 \%)$, non-CR/non-PD in $9(\mathrm{SIII}=5 / \mathrm{SIV}=4,33 \%)$, and $\mathrm{PD}$ in $4(\mathrm{SIII}=1 / \mathrm{SIV}=3,15 \%)$ patients. Patients with PD proceeded to CRS/HIPEC when there was evidence of reduction to bulky disease and complete cytoreduction was deemed feasible, despite evidence of new lesions after NACT.

Disease burden was also measured radiographically by extent of remaining omental disease, volume of ascites/pleural effusion, and lesion size pre/post-NACT. Reduction/resolution in each parameter was seen in $91 \%, 91 \%, 100 \%$, and $67 \%$ SIII and $85 \%, 92 \%, 71 \%$, and $92 \%$ SIV, respectively (Table 1 ).

3.2. Interval CRS/HIPEC Characteristics. Intraoperative characteristics are described in Table 2. Bowel anastomoses (colorectal, small bowel, ileocolonic, and colocolonic) were performed in $22(81 \%)$ with $13(48 \%)$ requiring 1 anastomosis $(\mathrm{SIII}=5 / \mathrm{SIV}=8)$ and $9(33 \%)$ requiring 2 anastomoses $(\mathrm{SIII}=4 /$ SIV $=5)$. Ostomy creation was not required in any patient.

Complete cytoreduction (CC-0/CC-1) was achieved in $11(92 \%)$ SIII and 15 (100\%) SIV patients. Patients with CC-1 $(n=4, \quad 15 \%, \quad$ SIII $=3 /$ SIV $=1)$ had residual nodules $(<0.25 \mathrm{~cm})$ in the mesentery, small bowel, or right upper quadrant. One SIII patient underwent incomplete cytoreduction (CC-2) with a sheath of scar tissue on the small bowel mesentery and distal ileum suspicious for disease that could not be completely excised.

HIPEC perfusion agents included carboplatin $(n=24$, $89 \%$, SIII $=12 / \mathrm{SIV}=12)$ and melphalan $(n=3,11 \%, \operatorname{SIV}=3)$. Median length of surgery was 8 hours in both groups. Median estimated blood loss was $425 \mathrm{~mL}$ SIII and $600 \mathrm{~mL}$ SIV $(p=0.170)$. Intraoperative blood transfusions were required in 7 (58\%) SIII and 9 (60\%) SIV; median: 2 units (range: $1-3$ ) in both groups. Postoperatively, 7 (58\%) SIII and 14 (93\%) SIV required transfusions ( $p=0.06$ ); median: 2 units (range: $1-4)$. Median ICU stay was 1 day in both groups. Median hospital stay was 8 days in SIII and 11 days in SIV $(p=0.01)$. All cases were high-grade serous carcinoma.

3.3. Postoperative Characteristics. Grade III/IV surgical complications occurred in $3(20 \%)$ SIV, including reoperation for wound dehiscence ( $n=1$, POD 21), indwelling thorax catheter for pleural effusion ( $n=1$, POD 27), and pneumothorax ( $n=1$, POD 1) (Table 2). Postoperatively, all patients were anemic. Leukopenia was seen in 3 (25\%) SIII and 7 SIV $(47 \%)(p=0.42)$. Thrombocytopenia was seen in 7 (58\%) SIII and $12(80 \%)$ SIV $(p=0.39)$. Twelve required granulocyte colony-stimulating factor agents $(44 \%$, SIII $=5 / \mathrm{SIV}=7)$. Median CA-125 post-CRS/HIPEC was $17 \mathrm{U} / \mathrm{mL}$ in SIII and $13 \mathrm{U} / \mathrm{mL}$ in SIV patients. Median sampled lymph nodes were 20 (IQR: 3-29). Seventeen patients $(65 \%$, SIII $=7 /$ SIV $=10)$ had positive lymph nodes.

Eleven (92\%) SIII and 10 (67\%) SIV received ASC, and median time to chemotherapy was 53 and 74 days, respectively $(p=0.007)$. Median ASC was 3 cycles (range: $2-6)$. SIII received $2(n=2)$ and $3(n=9)$ cycles versus SIV who received $2(n=1), 3(n=7)$, and $4(n=2)$ cycles. Indications for no ASC were 6 NACT cycles requiring maintenance bevacizumab $(n=3)$, patient declined $(n=2)$, and failure to thrive $(n=1)$. Median CA-125 after ASC was $11 \mathrm{U} / \mathrm{mL}$ SIII and $9 \mathrm{U} / \mathrm{mL}$ SIV with abnormal levels noted in one SIII patient $(40.6 \mathrm{U} / \mathrm{mL})$ (Table 2).

3.4. Recurrence. Nine (81\%) SIII and 12 (80\%) SIV recurred after a median of 12 (IQR: 10.4-13) and 11 (IQR: 8.5-18.6) months, respectively. Disease recurrence limited to one region occurred in 12 patients: 5 abdominopelvic (SIII $=3$ / SIV =2), 6 lymph nodes (SIII $=2 / \mathrm{SIV}=4)$, and 1 distant site (nonabdominopelvic cavity/nonlymph node) $(\mathrm{SIV}=1)$. Recurrence in multiple regions occurred in 10 patients: 3 abdominopelvic/lymph nodes (SIII $=2 / \mathrm{SIV}=1), 2$ abdominopelvic/distant site $(\mathrm{SIII}=1 / \mathrm{SIV}=1), 3 \mathrm{lymph}$ node/distant site $(\mathrm{SIII}=1 / \mathrm{SIV}=2)$, and 2 abdominopelvic/lymph node/ distant site $(\mathrm{SIII}=1 / \mathrm{SIV}=1)$. Sites of distant metastases were thorax $(n=6)$ and liver $(n=2)$ (Table 3$)$.

Of 11 (92\%) SIII ASC patients, $9(82 \%)$ recurred after a median time of 9 months (IQR: 7-10) from the last ASC cycle and 12 months (IQR: 10-12) from CRS/HIPEC. One SIII without ASC recurred 8 months after CRS/HIPEC. Of $10(67 \%)$ SIV ASC patients, 9 recurred after a median time of 11 months (IQR: 6-21) from the last ASC cycle and 16 months (IQR: 10-24) from CRS/HIPEC. Of 4 SIV patients without ASC, 3 recurred after 3, 7, and 9 months after CRS/ HIPEC, and 1 died of another cause.

3.5. Survival and Progression-Free Survival. Overall survival at 1,2 , and 3 years was $92.6 \%, 79.4 \%$, and $45.1 \%$, respectively; median: OS 33 months. PFS at 1, 2, and 3 years was $50 \%, 21.2 \%$, and $10.6 \%$, respectively; median PFS: 11.8 months.

Five (42\%) SIII and 10 (67\%) SIV patients were alive after a median follow-up of 31 and 30 months, respectively. OS at 1,2 , and 3 years was $100 \%, 83.3 \%$, and $33.3 \%$ in SIII and $86.7 \%, 75.8 \%$, and $56.9 \%$ in SIV, respectively. Median OS was 25 and 51 months in SIII and SIV, respectively $(p=0.269)$. One and 2 years PFS was $54.4 \%$ and $18.2 \%$ in SIII and $46.7 \%$ and $23.3 \%$ in SIV, respectively. Median PFS was 12.4 and 11.5 months in SIII and SIV, respectively $(p=0.944)$ (Figure 2). 


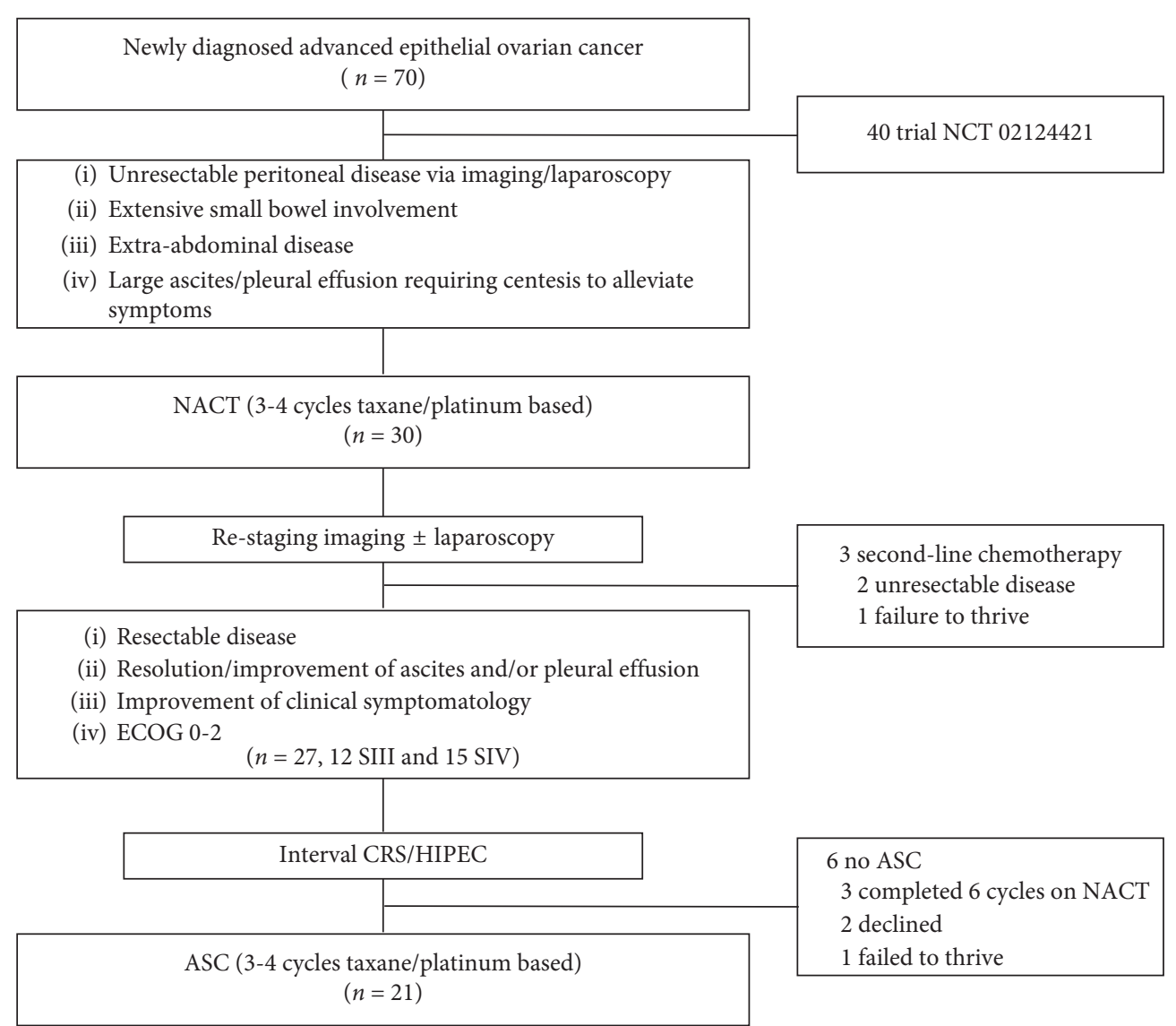

FIGURE 1: Management algorithm of initially unresectable stage IIIC and IV ovarian cancer patients. ASC: Adjuvant systemic chemotherapy, CRS/HIPEC: Cytoreductive surgery and hyperthermic intraperitoneal chemotherapy, ECOG: Eastern Cooperative Oncology Group Performance Status, NACT: Neoadjuvant systemic chemotherapy, SIII: Stage IIIC, SIV: Stage IVA/B.

TABLE 1: Characteristics of epithelial ovarian cancer patients with neoadjuvant chemotherapy.

\begin{tabular}{|c|c|c|c|c|}
\hline Characteristic & All population $(n=27)$ & Stage III $(n=12)$ & Stage IV $(n=15)$ & $p$ value \\
\hline \multicolumn{5}{|l|}{ Pre-NACT } \\
\hline Median age at diagnosis [IQR], years & $65[59-70]$ & $64[58-65]$ & $66[62-74]$ & 0.249 \\
\hline Median body mass index [IQR], $\mathrm{kg} / \mathrm{m}^{2}$ & $26[23.4-29.7]$ & $28.2[22-32.7]$ & $25.9[23.4-28.6]$ & 0.354 \\
\hline Germline $B R C A 1 / 2$ mutation, $n(\%)$ & $1 / 19(5)$ & $1 / 8(13)$ & $0 / 11(0)$ & - \\
\hline \multicolumn{5}{|l|}{ Procedures with $(+)$ cytology, $n(\%)$} \\
\hline Paracentesis & $15 / 15(100)$ & $10 / 10(100)$ & $5 / 5(100)$ & - \\
\hline Thoracentesis & $6 / 6(100)$ & $0(0)$ & $6 / 6(100)$ & - \\
\hline Median volume of paracentesis [IQR], mL & $3000[1600-5600]$ & $3500[1500-6025]$ & $2600[1450-4550]$ & - \\
\hline Median volume of thoracentesis [IQR], mL & $1500[950-1700]$ & NA & $1500[950-1700]$ & - \\
\hline \multicolumn{5}{|l|}{ NACT ${ }^{*}$} \\
\hline Median time from diagnosis to NACT [IQR], days & $26[19-33]$ & $29[20-34]$ & $25[15-36]$ & 0.680 \\
\hline Median NACT cycles, (range) & $3(3-6)$ & $3(3-4)$ & $4(3-6)$ & 0.080 \\
\hline \multicolumn{5}{|l|}{ Cycles of NACT, $n(\%)$} \\
\hline 3 cycles & $16(59)$ & $9(75)$ & $7(47)$ & - \\
\hline 4 cycles & $7(26)$ & $3(25)$ & $4(27)$ & - \\
\hline 6 cycles $\dagger$ & $4(15)$ & $0(0)$ & $4(27)$ & - \\
\hline \multicolumn{5}{|l|}{ NACT response } \\
\hline \multicolumn{5}{|l|}{ Hypoalbuminemia $\neq, n(\%)$} \\
\hline Before NACT & $10(37)$ & $5(41.7)$ & $5(33.3)$ & 0.656 \\
\hline After NACT & $1(4)$ & $0(0.0)$ & $1(6.7)$ & 1.000 \\
\hline \multicolumn{5}{|l|}{ Median CA-125 [IQR], U/mL } \\
\hline Before NACT & 1077 [500-3317] & 993 [297-3124] & 1168 [707-3460] & 0.661 \\
\hline After NACTI & 20.4 [11-69] & $21[12-68]$ & $19[8-70]$ & 0.464 \\
\hline
\end{tabular}


TABLE 1: Continued.

\begin{tabular}{|c|c|c|c|c|}
\hline Characteristic & All population $(n=27)$ & Stage III $(n=12)$ & Stage IV $(n=15)$ & $p$ value \\
\hline \multicolumn{5}{|l|}{ Radiographic response to NACT, $n(\%)$} \\
\hline Reduction or resolved, omental caking & $21 / 24(88)$ & $10 / 11(91)$ & $11 / 13(85)$ & - \\
\hline Reduction or resolved, ascites & $21 / 23(91)$ & $10 / 11(91)$ & $11 / 12(92)$ & - \\
\hline Reduction or resolved, pleural effusion & $9 / 11(82)$ & $4 / 4(100)$ & $5 / 7(71)$ & - \\
\hline Reduction or resolved, lesions & $18 / 21(86)$ & $6 / 9(67)$ & $12 / 13(92)$ & - \\
\hline
\end{tabular}

${ }^{*}$ All patients received taxane/platinum chemotherapy with bevacizumab added in 1 patient. $\dagger$ Four SIV patients received 6 cycles due to physician preference $(n=3)$ and coronary artery stent placement postponing surgery $(n=1)$. $¥$ Serum albumin levels $<3.5 \mathrm{~g} / \mathrm{dL}$. IFive ( $42 \%)$ SIII and 6 (40\%) SIV had abnormal CA125 levels $(>35 \mathrm{U} / \mathrm{mL}$ ) Post-NACT (range $=36-228 \mathrm{U} / \mathrm{mL}$ ). IQR: interquartile range; NACT: neoadjuvant chemotherapy.

TABLE 2: CRS/HIPEC and postoperative characteristics by stage.

\begin{tabular}{|c|c|c|c|c|}
\hline Characteristic & Total population $(n=27)$ & Stage III $(n=12)$ & Stage IV $(n=15)$ & $p$ value \\
\hline \multicolumn{5}{|l|}{ CRS/HIPEC characteristics } \\
\hline Median time from NACT to CRS/HIPEC [IQR], days & $40[35-45]$ & $41[30-47]$ & $40[35-45]$ & 0.757 \\
\hline Median PCI [IQR] & $20[12-27]$ & $21[10-29]$ & $20[13-26]$ & 0.926 \\
\hline \multicolumn{5}{|l|}{ Extent of resections, median $[\mathrm{IQR}]$} \\
\hline Organs resected & $7[5-8]$ & $6[5-8]$ & $7[6-8]$ & 0.490 \\
\hline Visceral peritonectomies & $2[2-3]$ & $2[1-3]$ & $2[2-3]$ & 0.409 \\
\hline Parietal peritonectomies & $4[3-4]$ & $4[3-4]$ & $4[3-5]$ & 0.504 \\
\hline Pelvic mass, $n(\%)$ & $24(89)$ & $9(75)$ & $15(100)$ & 0.075 \\
\hline \multicolumn{5}{|l|}{ Bowel anastomosis, $n(\%)$} \\
\hline 0 & $5(19)$ & $3(25)$ & $2(13)$ & - \\
\hline 1 & $13(48)$ & $5(42)$ & $8(53)$ & - \\
\hline 2 & $9(33)$ & $4(33)$ & $5(33)$ & - \\
\hline \multicolumn{5}{|l|}{ CC score, $n(\%)$} \\
\hline CC-0 (no visible tumor) & $22(81)$ & $8(67)$ & $14(93)$ & - \\
\hline CC-1 $($ tumor $\leq 0.25 \mathrm{~cm})$ & $4(15)$ & $3(25)$ & $1(7)$ & - \\
\hline CC-2 (tumor $0.25 \mathrm{~cm}-2.5 \mathrm{~cm}$ ) & $1(4)$ & $1(8)$ & $0(0)$ & - \\
\hline Median length of surgery [IQR], hours & $8[7-8]$ & $8.3[6.4-9.3]$ & $8[7-8.3]$ & 0.482 \\
\hline Estimated blood loss [IQR], mL & $500[350-800]$ & $425[275-600]$ & $600[350-1000]$ & 0.170 \\
\hline \multicolumn{5}{|l|}{ Transfusions, $n(\%)$} \\
\hline Intraoperative & $16(59)$ & $7(58)$ & $9(60)$ & 1.000 \\
\hline Postoperative & $21(78)$ & $7(58)$ & $14(93)$ & 0.060 \\
\hline Median length of hospitalization [IQR], days & $9[7-11]$ & $8[6-9]$ & $11[8-12]$ & 0.010 \\
\hline \multicolumn{5}{|l|}{ Tumor site, $n(\%)$} \\
\hline Ovary & $9(33)$ & $4(33)$ & $5(33)$ & - \\
\hline Fallopian tube & $11(41)$ & $4(33)$ & $7(47)$ & - \\
\hline Primary peritoneal & $7(26)$ & $4(33)$ & $3(20)$ & - \\
\hline \multicolumn{5}{|l|}{ Postoperative characteristics } \\
\hline Grade III/IV surgical complications, $n(\%)$ & $3(11)$ & $0(0)$ & $3(23)$ & - \\
\hline \multicolumn{5}{|l|}{ Hematologic toxicity, $n(\%)$} \\
\hline \multicolumn{5}{|l|}{ Anemia } \\
\hline Preoperative & $22(82)$ & $10(83)$ & $11(73)$ & 0.662 \\
\hline Postoperative & $27(100)$ & $12(100)$ & $15(100)$ & - \\
\hline \multicolumn{5}{|l|}{ Leukopenia } \\
\hline Preoperative & $10(37)$ & $6(50)$ & $4(27)$ & 0.257 \\
\hline Postoperative & $10(37)$ & $3(25)$ & $7(47)$ & 0.424 \\
\hline \multicolumn{5}{|l|}{ Thrombocytopenia } \\
\hline Preoperative & $1(4)$ & $0(0)$ & $1(7)$ & 1.000 \\
\hline Postoperative & $19(70)$ & $7(58)$ & $12(80)$ & 0.398 \\
\hline Median CA-125 post-CRS/HIPEC [IQR] $(n), \mathrm{U} / \mathrm{mL}$ & $13[8.2-23.2]$ & $17[8.2-28.6](10)$ & 13 [7.3-21.9] (12) & 0.767 \\
\hline Positive lymph nodes, $n(\%)$ & $17 / 26(65)$ & $7 / 11(63)$ & $10(67)$ & 0.706 \\
\hline Patients with ASC, $n(\%)$ & $21(78)$ & $11(92)$ & $10(67)$ & 0.182 \\
\hline Median time from CRS/HIPEC to ASC [IQR], days & $57[51-73]$ & $53[40-58]$ & $74[56-91]$ & 0.007 \\
\hline Median CA-125 post-ASC [IQR] $(n), \mathrm{U} / \mathrm{mL}$ & $9.5[7-13]$ & $11[7-18.2](11)$ & $9[7.3-12.3](8)$ & 0.385 \\
\hline Recurrence, $n(\%)$ & $21(81)$ & $9 / 11(81)$ & $12(80)$ & 0.236 \\
\hline Alive, $n(\%)$ & $15(55)$ & $5(42)$ & $10(67)$ & - \\
\hline Median follow-up (95\% CI), months & $31[22-40]$ & $31[23-39]$ & $30[14-46]$ & - \\
\hline
\end{tabular}

ASC: adjuvant systemic chemotherapy, CC: completeness of cytoreduction score, CI: confidence interval, CRS/HIPEC: cytoreductive surgery and hyperthermic intraperitoneal chemotherapy, IQR: interquartile range, NACT: neoadjuvant chemotherapy. 


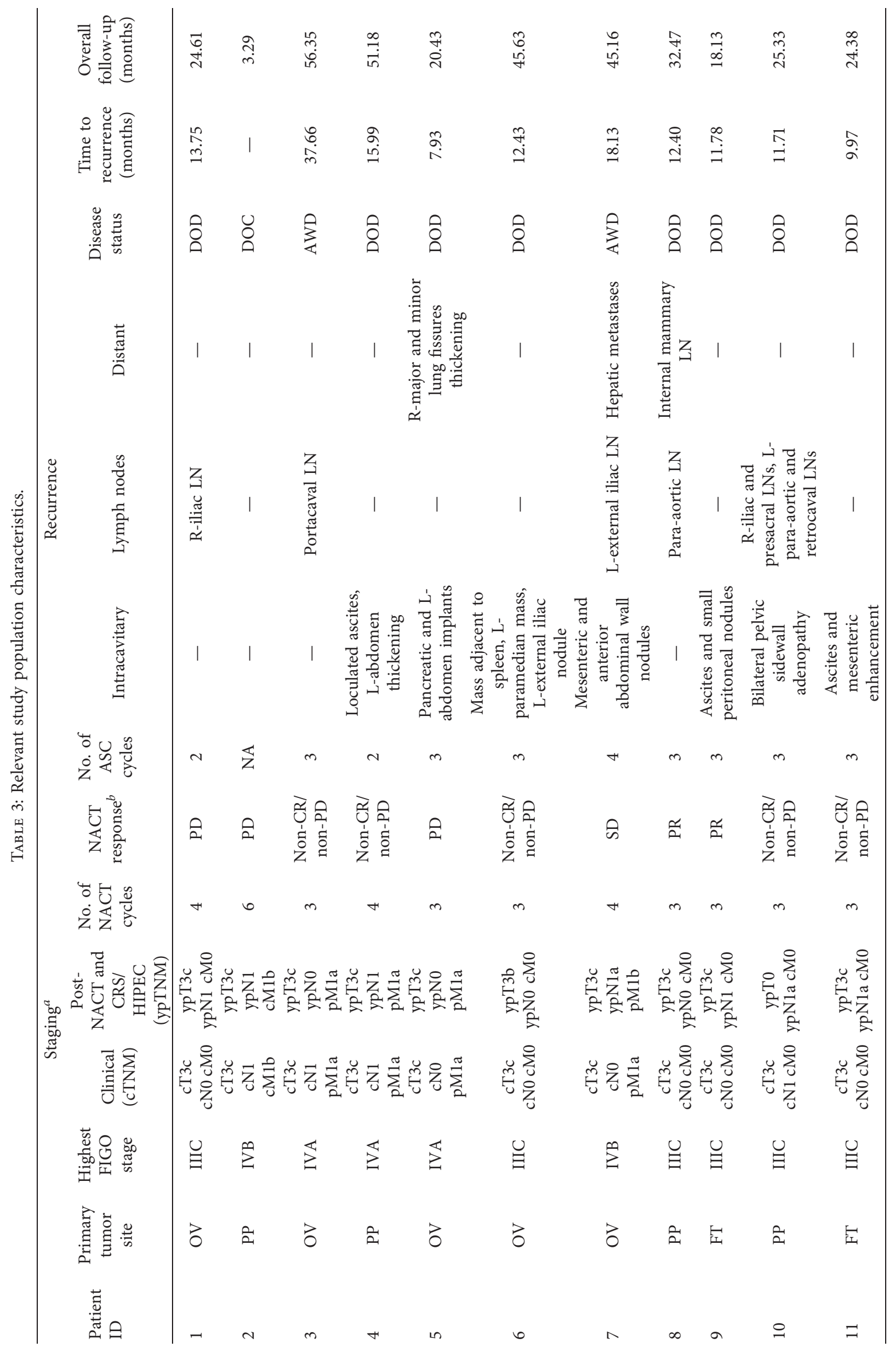




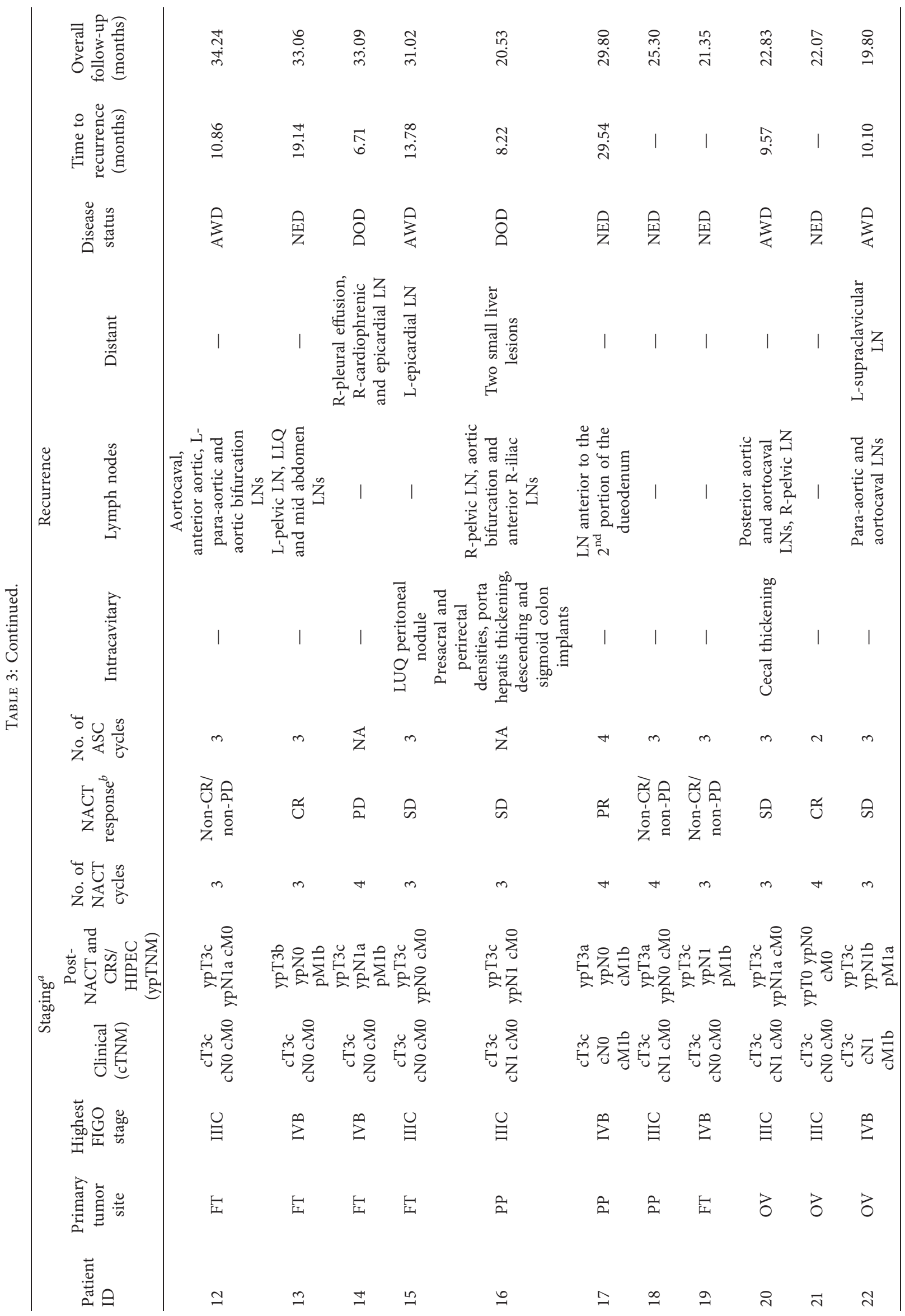




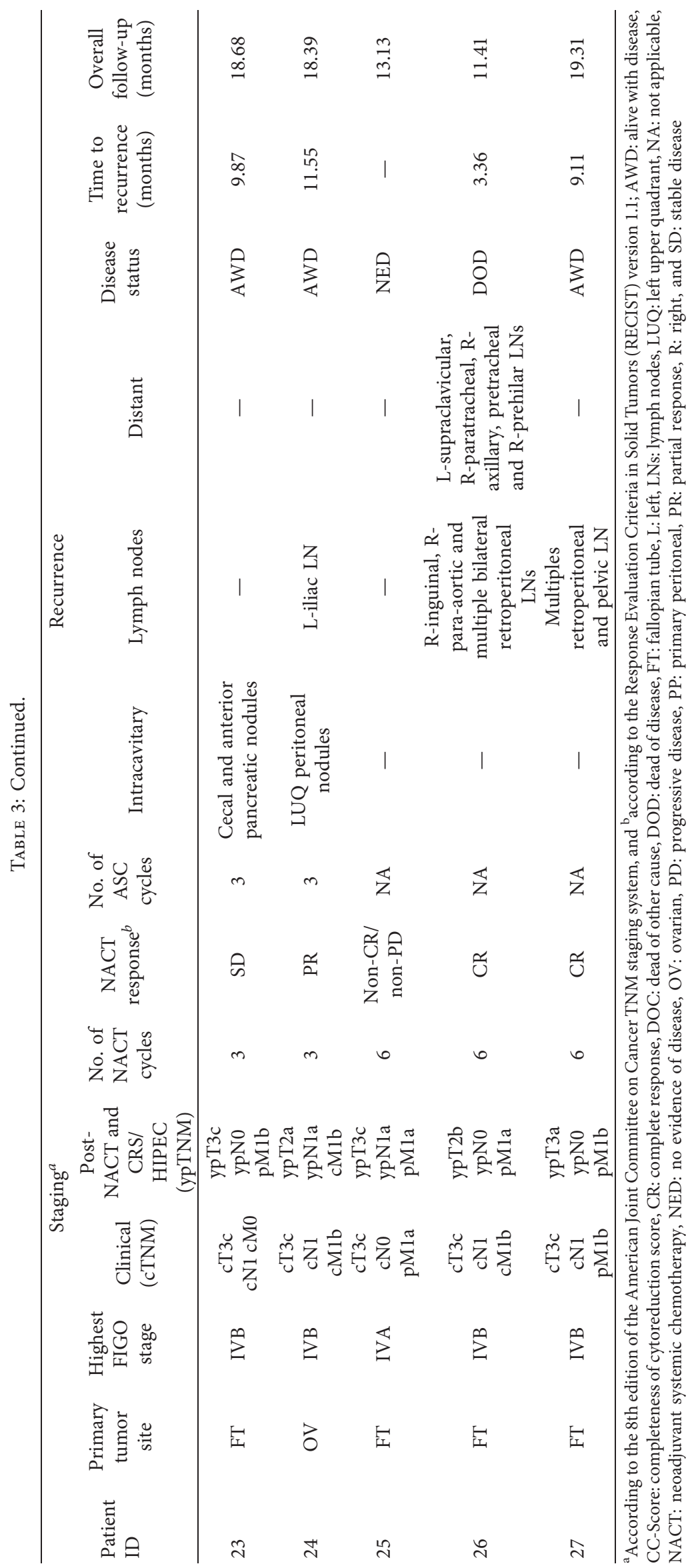



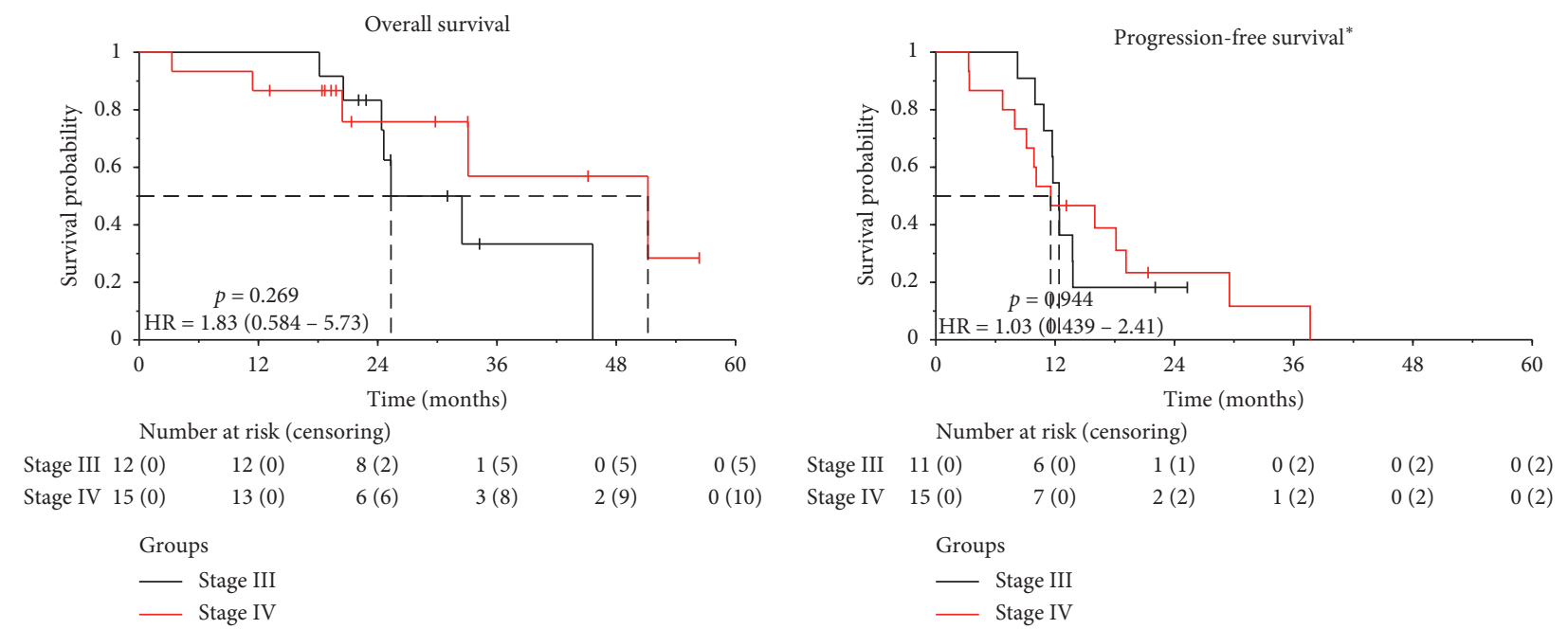

FIgURE 2: Overall survival and progression-free survival by disease stage in patients with peritoneal metastases from epithelial ovarian cancer treated with neoadjuvant systemic chemotherapy followed by cytoreductive surgery and hyperthermic intraperitoneal chemotherapy. ${ }^{\star}$ Progression-free survival was only calculated in patients with CC-0 or CC-1. HR: hazard ratio.

\section{Discussion}

Newly diagnosed FIGO SIV ovarian cancer patients who initially present with extensive, unresectable disease, or poor performance status are typically managed with a palliative approach. However, NACT followed by interval CRS/HIPEC can offer therapeutic benefit for these advanced patients. In our study, SIV had similar outcomes to and a tendency for improved cytoreduction rates (CC-0: 67\% vs. 93\%) and survival (median OS: 25 vs. 51 months) than SIII.

NACT prior to surgical debulking is considered under the premise that reducing tumor burden will improve patient performance and increase complete cytoreduction rates, resulting in lower morbidity, shorter hospital stay, and improved quality of life $[10,21-23]$. However, no randomized trial has demonstrated superior survival of NACT plus interval debulking to primary debulking surgery ( $\mathrm{Ta}-$ ble 4) [7-11, 24]. Moreover, the dominant factor influencing AEOC survival is the quality of cytoreduction, with $>2 \mathrm{~cm}$ residual disease providing no survival benefit [4, 25-28]. Thus, NACT plus interval debulking has been reserved only for patients who are not surgical candidates, commonly due to pleural disease, massive ascites, or extensive small bowel involvement. Interestingly, despite the controversial benefit of NACT in a broad AEOC patient cohort, pooled analysis demonstrates that NACT offers better survival in SIV disease with high tumor burden or poor performance status [5]. In our study, 90\% (27/30) who underwent NACT became surgical candidates achieving 96\% complete cytoreduction rate and 9-day median hospital stay with encouraging cytoreduction rates and survival outcomes experienced in 15 SIV patients compared to SIII.

Traditionally, NACT is combined with standard debulking surgery; however, the addition of HIPEC during interval debulking surgery is gaining interest $[6,29,30]$. HIPEC has promising results treating other cancers that commonly present with peritoneal spread [31-33]. HIPEC allows for direct contact of high-dose, locoregional chemotherapy, potentiated by heat increasing cytotoxicity and tissue penetration, inhibiting angiogenesis, and inducting apoptosis [34-37]. Even after complete removal of macroscopic disease, microscopic tumor cells likely remain and could contribute to early recurrence rates [38]. This may especially be true after NACT where both complete and partial response to therapy can appear as scar tissue intraoperatively [39]. Therefore, the addition of HIPEC may offer improved outcomes for these patients.

Although taxane and platinum agents are commonly used with remarkable hyperthermic effects, further investigation into the optimal HIPEC agents is needed, especially in platinum/chemo-resistant disease $[40,41]$. The majority of our patients $(88 \%)$ received carboplatin. However, those with seemingly platinum-resistant disease requiring 6 NACT cycles $(n=3)$ were given melphalan, an alternative HIPEC agent for aggressive and recurrent peritoneal malignancies [42, 43].

Previous studies report the benefits of HIPEC for recurrent AEOC. Spiliotis et al. conducted the first prospective, randomized phase III study investigating HIPEC as an alternative treatment in recurrent stage IIIC/IV AEOC after PDS and systemic chemotherapy [44]. Patients underwent CRS/HIPEC $(n=60)$ or CRS alone $(n=60)$, both followed by ASC. PCI was $\geq 10$ in $48 \%$ and $50 \%$ and CC- 0 was achieved in $65 \%$ and $55 \%$, by treatment group, respectively, with the HIPEC having significantly longer OS (median OS: 27 versus 13 months $(p=0.006))$. Despite the fact that these results are controversial, subanalysis by stage was not performed, and our cohort relates only to initially diagnosed patients; data from this first trial suggests that HIPEC may be beneficial in recurrent SIV AEOC [45].

Van Driel et al. published the results of their randomized phase III trial in SIII EOC comparing NACT and CRS/HIPEC $(n=122)$ vs. CRS alone $(n=123)$, both followed by ASC [6]. Disease burden was measured by the number of abdominal 


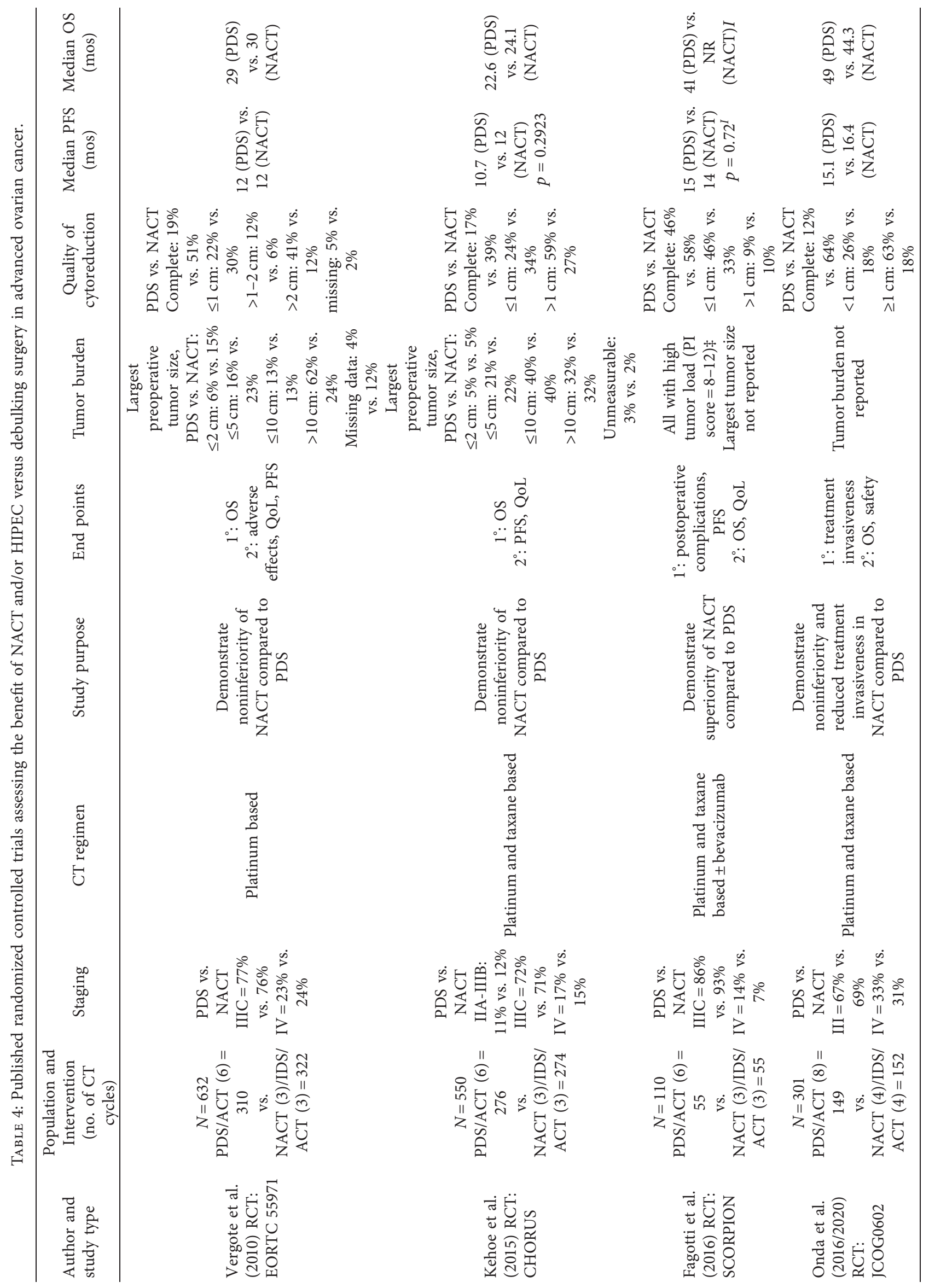




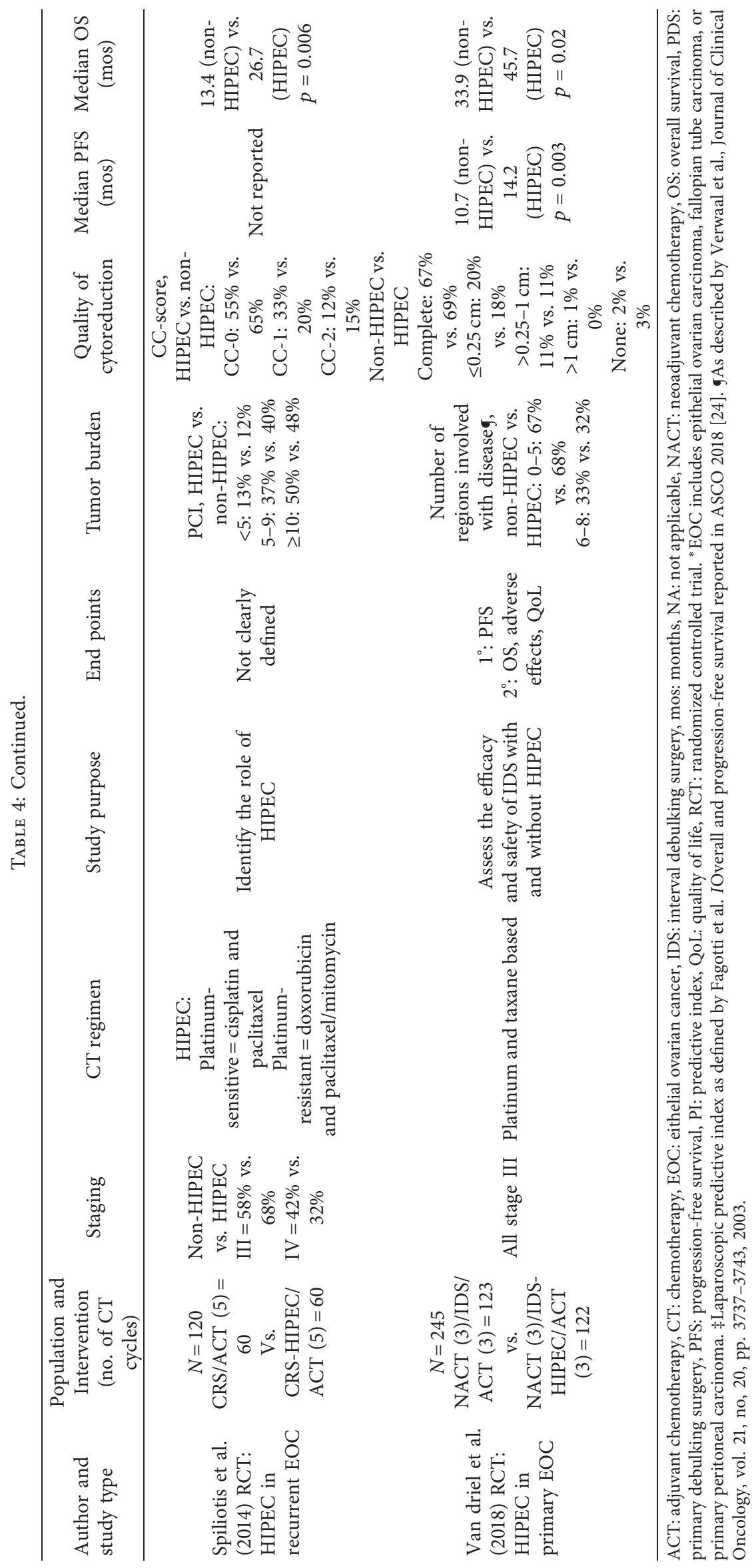


regions involved, rather than $\mathrm{PCI}$, making comparisons to other HIPEC studies inequitable [6, 44]. Nevertheless, in HIPEC versus no-HIPEC groups, 6-8 regions were involved in $32 \%$ and $33 \%$, with CC-0 achieved in $69 \%$ and $67 \%$, respectively. Median OS was 46 versus 34 months $(p=0.02)$, respectively, without significant differences in postoperative complications or health-related quality of life. Comparatively, our study included only initially unresectable patients including both SIII and SIV disease. Disease burden was extensive in our SIV patients (median PCI: 20) and CC-0 achieved in $93 \%$, with median OS of 51 months.

Despite variations of reported OS, HIPEC consistently demonstrated improved survival compared to controls (Table 4) $[6,29,44]$. Variances could be explained by differences in disease burden, complete cytoreduction rates, or quality of surgery. Spiliotis et al. reported a shorter median OS (27 months) for recurrent SIII/IV compared to Van Driel et al. who reported 46 months median OS with NACT and interval CRS/HIPEC in SIII patients. However, the Spiliotis et al. study had higher disease burden and lower cytoreduction rates [44]. In our cohort, SIII and SIV intraoperative median PCI was 21 and 20, CC-0 was achieved in $67 \%$ and $93 \%$, and median OS was 25 months and 51 months, respectively. Our population presented with high disease burden, even after NACT, and this may represent aggressive tumor biology although maximum surgical effort and high complete cytoreduction rates were achieved through combined surgical efforts of gynecologic and surgical oncologists. Regardless, SIV NACT and interval CRS/ HIPEC patients in our cohort demonstrated comparable outcomes to unresectable SIII.

The absence of ostomies in our cohort highlights the collaboration between gynecologic and surgical oncologists. This can be compared to the $72 \%$ ostomy rate in the Van Driel et al. trials' CRS/HIPEC group and among other reports ranging from 17-97\% after AEOC debulking surgery $[6,46,47]$. We were able to avoid ostomy creation despite single and double bowel anastomosis in $48 \%$ and $33 \%$, respectively. Surgeons should be cognizant that performing bowel resections during CRS/HIPEC does not necessitate ostomy creation. Surgical oncologists are highly skilled and experienced in performing bowel resections with anastomoses. This enhances the collaborative effort between gynecologic and surgical oncology, translating to improved surgical outcomes and patient quality of life.

In our study, overall time from CRS/HIPEC to ASC was significantly longer compared to Van Driel trial (median time of 57 days versus 33 days, respectively). Nevertheless, median length of surgery was considerably longer in our cohort (8 versus 5.6 hours) suggesting extensive resections to achieve complete cytoreductions in patients with extensive disease. Disease burden and quality of cytoreduction play a role in the delay of ASC after CRS/HIPEC due to longer recovery time with extensive procedures and multiple organ resections.

We also found that median hospital stay and time to ASC were significantly longer in SIV than in SIII. Review of cases revealed that more than half of SIV patients had a hospital stay $\geq 10$ days ( $60 \%$ SIV vs. $8 \%$ SIII) with median PCI of 24 when hospital stay exceeded 10 days vs. median PCI of 19 when hospital stay was $<10$ days $(p=0.19)$. Furthermore, these SIV patients also had the longest time to ASC ( $>8$ weeks) of which delays to adjuvant therapy were often patient driven. Thus, it seems that SIV patients with high PCI may require longer hospital stay and more time recovering before resuming chemotherapy.

Study limitations include its retrospective design at a single institution, small sample size, and limited follow-up. However, it includes detailed perioperative characteristics and outcomes of SIV disease, a subgroup that has shown to benefit the most from multimodal treatment approaches, such as NACT plus interval debulking surgery and warrant further studies [5]. SIV patients were excluded from the Van Driel et al. trial; nonetheless, these results and other reports are encouraging to further explore the role of NACT and interval CRS/HIPEC in SIV disease [6, 14, 29].

\section{Conclusions}

Promising survival outcomes, similar to stage IIIC, can be achieved for patients with initially unresectable, stage IV EOC treated with NACT and interval CRS/HIPEC. Randomized studies are needed to assess the long-term outcomes of NACT plus CRS/HIPEC in stage IV ovarian cancer.

\section{Abbreviations}

AEOC: Advanced epithelial ovarian, fallopian tube, and primary peritoneal cancers

ASC: Adjuvant systemic chemotherapy

CC: $\quad$ Completeness of cytoreduction

CR: Complete response

CRS: Cytoreductive surgery

CT: $\quad$ Computerized tomography

ECOG: Eastern cooperative oncology group

HIPEC: Hyperthermic intraperitoneal chemotherapy

ICU: Intensive care unit

IQR: Interquartile range

LMWH: Low-molecular weight heparin

NACT: Neoadjuvant systemic chemotherapy

OS: Overall survival

PCI: Peritoneal cancer Index

PDS: $\quad$ Primary debulking surgery

PFS: $\quad$ Progression-free survival

RECIST: Response evaluation criteria in solid tumors.

\section{Data Availability}

The data used to support the findings of this study are available from the corresponding author upon request.

\section{Conflicts of Interest}

The authors declare that there are no conflicts of interest regarding the publication of this paper.

\section{Authors' Contributions}

CAMZ was involved in the methodology, investigation, data curation, formal analysis, and visualization. AS was 
responsible for the conceptualization, methodology, and investigation. MS was involved in the project administration, investigation, data curation, formal analysis, and visualization. FLR was involved with data analysis and provided statistical assistance. VG was involved in the conceptualization and investigation. MCK investigated the study and curated the data. TPDM was responsible for the conceptualization, methodology, and investigation. CAMZ, AS, MS, VG, MCK, CN, FLR, and TPDM wrote, reviewed, and edited the original draft.

\section{Acknowledgments}

Research and publication are funded by the Internal Research Fund supported by patient donations.

\section{References}

[1] L. G. Frederick, L. P. David, and D. F. Irvin, AJCC Cancer Staging Manual, Springer International Publishing, New York, NY, USA, 8th edition, 2017.

[2] R. J. Kurman, M. L. Carcangiu, C. S. Herrington, and R. H. Young, "WHO classification of tumours," WHO Classification of Tumours of Female Reproductive Organs, p. 307, International Agency for Research on Cancer, Lyon, France, 2014.

[3] American Cancer Society, "Cancer facts \& figures 2019," 2019, https://www.cancer.org/cancer/ovarian-cancer/about/ key-statistics.html.

[4] NCCN, “Ovarian cancer (version 1.2019)," 2019, https://www. nccn.org/professionals/physician_gls/pdf/ovarian.pdf.

[5] I. Vergote, C. Coens, M. Nankivell et al., "Neoadjuvant chemotherapy versus debulking surgery in advanced tuboovarian cancers: pooled analysis of individual patient data from the EORTC 55971 and CHORUS trials," Lancet Oncology, vol. 19, no. 12, pp. 1687-1680, 2018.

[6] W. J. Van Driel, S. N. Koole, K. Sikorska et al., "Hyperthermic intraperitoneal chemotherapy in ovarian cancer," New England Journal of Medicine, vol. 378, no. 3, pp. 230-240, 2018.

[7] I. Vergote, C. G. Tropé, F. Amant et al., "Neoadjuvant chemotherapy or primary surgery in stage IIIC or IV ovarian cancer," New England Journal of Medicine, vol. 363, no. 10, pp. 943-953, 2010.

[8] A. Fagotti, G. Ferrandina, G. Vizzielli et al., "Phase III randomised clinical trial comparing primary surgery versus neoadjuvant chemotherapy in advanced epithelial ovarian cancer with high tumour load (SCORPION trial): final analysis of peri-operative outcome," European Journal of Cancer, vol. 59, pp. 22-33, 2016.

[9] S. Kehoe, J. Hook, M. Nankivell et al., "Primary chemotherapy versus primary surgery for newly diagnosed advanced ovarian cancer (CHORUS): an open-label, randomised, controlled, non-inferiority trial," The Lancet, vol. 386, no. 9990, pp. 249-257, 2015.

[10] T. Onda, T. Satoh, T. Saito et al., "Comparison of treatment invasiveness between upfront debulking surgery versus interval debulking surgery following neoadjuvant chemotherapy for stage III/IV ovarian, tubal, and peritoneal cancers in a phase III randomised trial: japan clinical oncology group study JCOG0602," European Journal of Cancer, vol. 64, pp. 22-31, 2016.

[11] T. Onda, T. Satoh, G. Ogawa et al., "Comparison of survival between primary debulking surgery and neoadjuvant chemotherapy for stage III/IV ovarian, tubal and peritoneal cancers in phase III randomised trial," European Journal of Cancer (Oxford, England: 1990), vol. 130, pp. 114-125, 2020.

[12] B. Kobal, M. Noventa, B. Cvjeticanin et al., "Primary debulking surgery versus primary neoadjuvant chemotherapy for high grade advanced stage ovarian cancer: comparison of survivals," Radiology and Oncology, vol. 52, no. 3, pp. 307-319, 2018.

[13] R. Warschkow, I. Tarantino, J. Lange et al., "Does hyperthermic intraoperative chemotherapy lead to improved outcomes in patients with ovarian cancer? A single center cohort study in 111 consecutive patients," Patient Safety in Surgery, vol. 6, no. 1, p. 12, 2012.

[14] F. C. Muñoz-Casares, F. J. Medina-Fernández, Á. ArjonaSánchez et al., "Peritonectomy procedures and HIPEC in the treatment of peritoneal carcinomatosis from ovarian cancer: long-term outcomes and perspectives from a high-volume center," European Journal of Surgical Oncology (EJSO), vol. 42, no. 2, pp. 224-233, 2016.

[15] E. A. Eisenhauer, P. Therasse, J. Bogaerts et al., "New response evaluation criteria in solid tumours: revised RECIST guideline (version 1.1)," European Journal of Cancer, vol. 45, no. 2, pp. 228-247, 2009.

[16] W. G. McCluggage, M. J. Judge, B. A. Clarke et al., "Data set for reporting of ovary, fallopian tube and primary peritoneal carcinoma: recommendations from the International collaboration on cancer reporting (ICCR)," Modern Pathology, vol. 28, no. 8, pp. 1101-1122, 2015.

[17] P. Jacquet and P. H. Sugarbaker, "Clinical research methodologies in diagnosis and staging of patients with peritoneal carcinomatosis," Cancer Treatment and Research, vol. 82, pp. 359-374, 1996.

[18] P. Sugarbaker, Technical Handbook for the Integration of Cytoreductive Surgery and Perioperative Intraperitoneal Chemotherapy into the Surgical Management of Gastrointestinal and Gynecologic Malignancy, Foundation for Applied Research in Gastrointestinal Oncology, Washington, DC, USA, 4th edition, 2005.

[19] P. A. Clavien, J. Barkun, M. L. de Oliveira et al., "The claviendindo classification of surgical complications," Annals of Surgery, vol. 250, no. 2, pp. 187-196, 2009.

[20] M. B. Amin and S. B. Edge, AJCC Cancer Staging Manual, Springer, Berlin, Germany, 2017.

[21] M. Baekelandt, "The potential role of neoadjuvant chemotherapy in advanced ovarian cancer," International Journal of Gynecologic Cancer, vol. 13, no. Suppl 2, pp. 163-168, 2003.

[22] J. Hou, M. Kelly, H. Yu et al., "Neoadjuvant chemotherapy lessens surgical morbidity in advanced ovarian cancer and leads to improved survival in stage IV disease," Gynecologic Oncology, vol. 105, no. 1, pp. 211-217, 2007.

[23] S. Kang and B.-H. Nam, "Does neoadjuvant chemotherapy increase optimal cytoreduction rate in advanced ovarian cancer? Meta-analysis of 21 studies," Annals of Surgical Oncology, vol. 16, no. 8, pp. 2315-2320, 2009.

[24] A. Fagotti, G. Vizzielli, G. Ferrandina et al., "Survival analyses from a randomized trial of primary debulking surgery versus neoadjuvant chemotherapy for advanced epithelial ovarian cancer with high tumor load (SCORPION trial)," Journal of Clinical Oncology, vol. 36, no. 15_suppl, p. 5516, 2018.

[25] D. G. Mutch, "Surgical management of ovarian cancer," Seminars in Oncology, vol. 29, no. 1, pp. 3-8, 2002.

[26] W. J. Hoskins, W. P. McGuire, M. F. Brady et al., "The effect of diameter of largest residual disease on survival after primary cytoreductive surgery in patients with suboptimal residual 
epithelial ovarian carcinoma," American Journal of Obstetrics and Gynecology, vol. 170, no. 4, pp. 974-980, 1994.

[27] C. W. E. Redman, J. Warwick, D. M. Luesley, R. Varma, F. G. Lawton, and G. R. P. Blackledge, "Intervention debulking surgery in advanced epithelial ovarian cancer," BJOG: An International Journal of Obstetrics and Gynaecology, vol. 101, no. 2, pp. 142-146, 1994.

[28] P. G. Rose, S. Nerenstone, M. F. Brady et al., "Secondary surgical cytoreduction for advanced ovarian carcinoma," New England Journal of Medicine, vol. 351, no. 24, pp. 2489-2497, 2004.

[29] Q. Wu, Q. Wu, J. Xu et al., "Efficacy of hyperthermic intraperitoneal chemotherapy in patients with epithelial ovarian cancer: a meta-analysis," International Journal of Hyperthermia, vol. 36, no. 1, pp. 562-572, 2019.

[30] M. L. Lopresti, C. A. Bandera, and T. J. Miner, "New approaches to improving survival after neoadjuvant chemotherapy: the role of intraperitoneal therapy and heated intraperitoneal chemotherapy in ovarian cancer," American Society of Clinical Oncology Educational Book, vol. 39, no. 39, pp. 19-23, 2019.

[31] W. Jimenez, A. Sardi, C. Nieroda et al., "Predictive and prognostic survival factors in peritoneal carcinomatosis from appendiceal cancer after cytoreductive surgery with hyperthermic intraperitoneal chemotherapy," Annals of Surgical Oncology, vol. 21, no. 13, pp. 4218-4225, 2014.

[32] T. D. Yan, M. Deraco, D. Baratti et al., "Cytoreductive surgery and hyperthermic intraperitoneal chemotherapy for malignant peritoneal mesothelioma: multi-institutional experience," Journal of Clinical Oncology, vol. 27, no. 36, pp. 6237-6242, 2009.

[33] C. A. Muñoz-Zuluaga, A. Sipok, and A. Sardi, "Management of peritoneal metastasis from uterine sarcoma," in Unusual Cases in Peritoneal Surface Malignancies, E. Canbay, Ed., Springer International Publishing, Cham, Switzerland, pp. 83-101, 2017.

[34] G. Panteix, A. Beaujard, F. Garbit et al., "Population pharmacokinetics of cisplatin in patients with advanced ovarian cancer during intraperitoneal hyperthermia chemotherapy," Anticancer Research, vol. 22, no. 2b, pp. 1329-1336, 2002.

[35] A. Sukovas, G. Silkuniene, S. Trumbeckaite et al., "Hyperthermia potentiates cisplatin cytotoxicity and negative effects on mitochondrial functions in OVCAR-3 cells," Journal of Bioenergetics and Biomembranes, vol. 51, no. 4, pp. 301-310, 2019.

[36] J. O. W. Pelz, M. Vetterlein, T. Grimmig et al., "Hyperthermic intraperitoneal chemotherapy in patients with peritoneal carcinomatosis: role of heat shock proteins and dissecting effects of hyperthermia," Annals of Surgical Oncology, vol. 20, no. 4, pp. 1105-1113, 2013.

[37] B. Zunino, C. Rubio-Patiño, E. Villa et al., "Hyperthermic intraperitoneal chemotherapy leads to an anticancer immune response via exposure of cell surface heat shock protein 90," Oncogene, vol. 35, no. 2, pp. 261-268, 2016.

[38] R. E. Bristow, R. S. Tomacruz, D. K. Armstrong, E. L. Trimble, and F. J. Montz, "Survival effect of maximal cytoreductive surgery for advanced ovarian carcinoma during the platinum era: a meta-analysis," Journal of Clinical Oncology, vol. 20, no. 5, pp. 1248-1259, 2002.

[39] E. J. M. Baltussen, S. G. Brouwer de Koning, J. Sanders et al., "Using diffuse reflectance spectroscopy to distinguish tumor tissue from fibrosis in rectal cancer patients as a guide to surgery," Lasers in Surgery and Medicine, vol. 52, no. 7, pp. 604-611, 2019.
[40] M. Muller, M. Chérel, P.-F. Dupré, S. Gouard, M. Collet, and J.-M. Classe, "Cytotoxic effect of hyperthermia and chemotherapy with platinum salt on ovarian cancer cells: results of an in vitro study," European Surgical Research, vol. 46, no. 3, pp. 139-147, 2011.

[41] T. Boissenot, A. Bordat, B. Larrat et al., "Ultrasound-induced mild hyperthermia improves the anticancer efficacy of both taxol and paclitaxel-loaded nanocapsules," Journal of Controlled Release, vol. 264, pp. 219-227, 2017.

[42] A. Sardi, W. Jimenez, C. Nieroda, M. Sittig, S. Shankar, and V. Gushchin, "Melphalan: a promising agent in patients undergoing cytoreductive surgery and hyperthermic intraperitoneal chemotherapy," Annals of Surgical Oncology, vol. 21, no. 3, pp. 908-914, 2014.

[43] A. Sardi, W. A. Jimenez, C. Nieroda, M. Sittig, R. Macdonald, and V. Gushchin, "Repeated cytoreductive surgery and hyperthermic intraperitoneal chemotherapy in peritoneal carcinomatosis from appendiceal cancer: analysis of survival outcomes," European Journal of Surgical Oncology (EJSO), vol. 39, no. 11, pp. 1207-1213, 2013.

[44] J. Spiliotis, E. Halkia, E. Lianos et al., "Cytoreductive surgery and HIPEC in recurrent epithelial ovarian cancer: a prospective randomized phase III study," Annals of Surgical Oncology, vol. 22, no. 5, pp. 1570-1575, 2015.

[45] P. Harter, A. Reuss, J. Sehouli, L. Chiva, and A. du Bois, "Brief report about the role of hyperthermic intraperitoneal chemotherapy in a prospective randomized phase 3 study in recurrent ovarian cancer from Spiliotis et al." International Journal of Gynecologic Cancer, vol. 27, no. 2, pp. 246-247, 2017.

[46] R. Tozzi, J. Casarin, A. Baysal et al., "Morbidity of multiple bowel resection compared to single bowel resection after debulking surgery for ovarian cancer," European Journal of Obstetrics \& Gynecology and Reproductive Biology, vol. 240, pp. 215-219, 2019.

[47] S. Mourton, L. Temple, N. Aburustum et al., "Morbidity of rectosigmoid resection and primary anastomosis in patients undergoing primary cytoreductive surgery for advanced epithelial ovarian cancer," Gynecologic Oncology, vol. 99, no. 3, pp. 608-614, 2005. 